\title{
Chemical Shift Detection with Energy Dispersive Spectroscopy (EDS)
}

\author{
Rebekah Jin ${ }^{1}$, Brian Zutter ${ }^{2}$, B. C. Regan ${ }^{3}$ and Matthew Mecklenburg ${ }^{4}$ \\ ${ }^{1}$ University of California, United States, ${ }^{2}$ University of California, Los Angeles, Los Angeles, California, \\ United States, ${ }^{3}$ Department of Physics and Astronomy, University of California, Los Angeles, California \\ 90095, United States, United States, ${ }^{4}$ The Aerospace Corporation, United States
}

The binding energies of an atom's core-state electrons are generally changed by chemical bonds, an effect called the chemical shift. A well-known example is the $\mathrm{L}_{2,3}$ edge in silicon, which changes from $99 \mathrm{eV}$ in pure silicon to $102.9 \mathrm{eV}$ in silicon nitride to $104 \mathrm{eV}$ in silicon dioxide [1]. When aluminum is oxidized, both its $\mathrm{L}_{2,3}$ and its K-edges shift up by $2-4 \mathrm{eV}$ from the $73 \mathrm{eV}$ characteristic of the pure metal [2,3]. With sub-1 eV energy resolution, electron energy loss spectroscopy (EELS) is commonly employed to detect these shifts, which are small compared to the binding energy. Here, we seek to develop a technique for measuring chemical shifts that is based on energy dispersive spectroscopy (EDS) instead.

When the full width at half-maximum (FWHM) of an x-ray peak is used as the metric, EDS has an energy resolution of order $100 \mathrm{eV}$ [4], which would make it useless for chemical shift analysis. But curve fitting can locate the center of a peak to much better precision than the FWHM. We perform EDS mapping on a lithographically defined, $100 \mathrm{~nm}$-thick aluminum nanowire supported by a 20 -nm thick silicon nitride membrane (Fig. 1A). The aluminum is coated with the usual native oxide. For each EDS spectrum in the datacube, we fit the aluminum K-alpha peak with a Gaussian (Fig. 1B). We are able to locate the EDS peak with a precision of $1 \mathrm{eV}$ or better, which is similar to the performance that can be achieved with nonmonochromated EELS.

We generate a map showing the $\mathrm{x}$-ray energy at each real-space position and apply a threshold to remove noise where no x-rays from the aluminum are detected (Fig. 2A). Averaging along the vertical (spatial) axis produces a line profile (Fig. 2B). Although no significant features are evident near the edge, a linear fit shows that the peak energy decreases by $0.8 \mathrm{eV}$ between the beginning and the end of the profile. While smaller than the 2 $\mathrm{eV}$ expected based on EELS results from the literature, the data show energy resolution that should be sufficient to detect chemical shifts. Though still ongoing, our work so far implies detectability; we are working to develop a sample optimized for demonstrating EDS-detectable chemical shifts. 

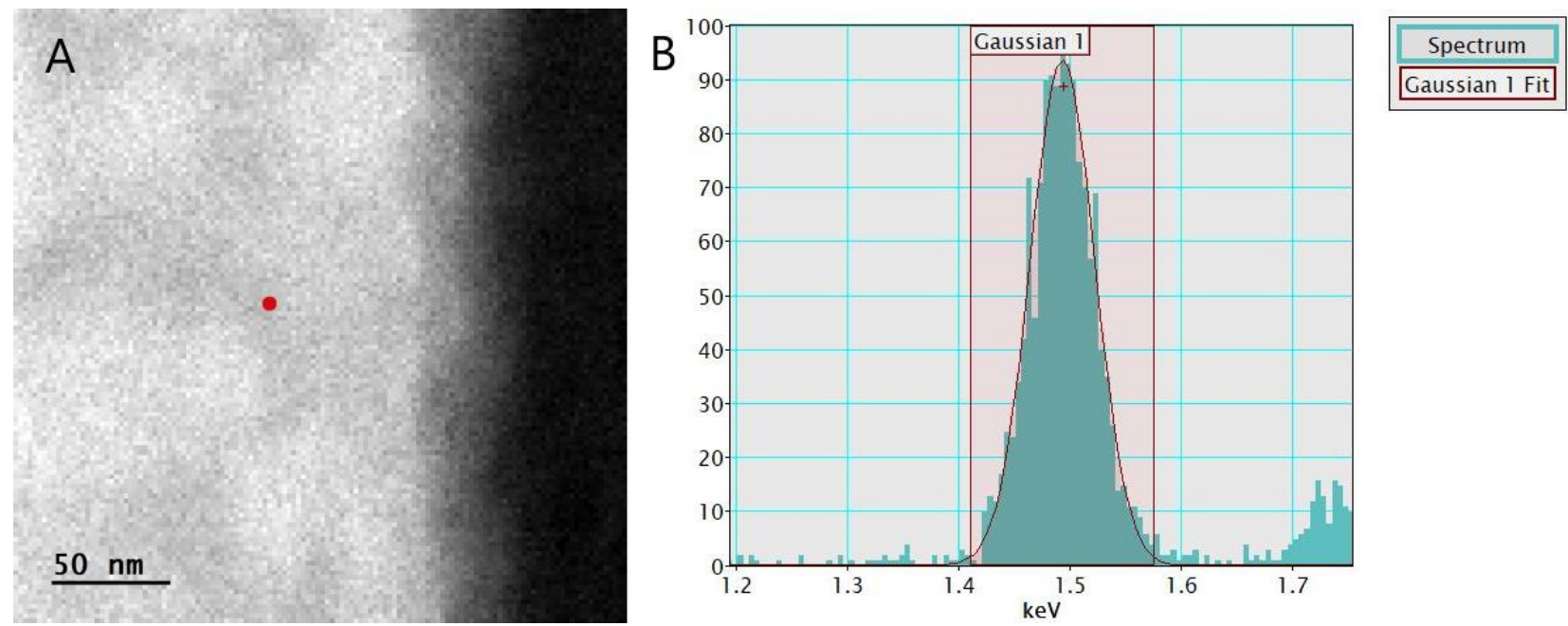

Figure 1. (A) An EDS spectrum image (summed in the energy direction) of a sample of aluminum about 100 $\mathrm{nm}$ thick on top of a $20 \mathrm{~nm}$ silicon nitride membrane. The red dot indicates the position at which the spectrum in (B) was taken. (B) The aluminum K-alpha line associated part of the EDS spectrum at the red pixel in (A) is fit to a Gaussian. The red background indicates the fit window. Gaussians are fit to all aluminum peaks across the image, then used to generate a map of the peak center positions.
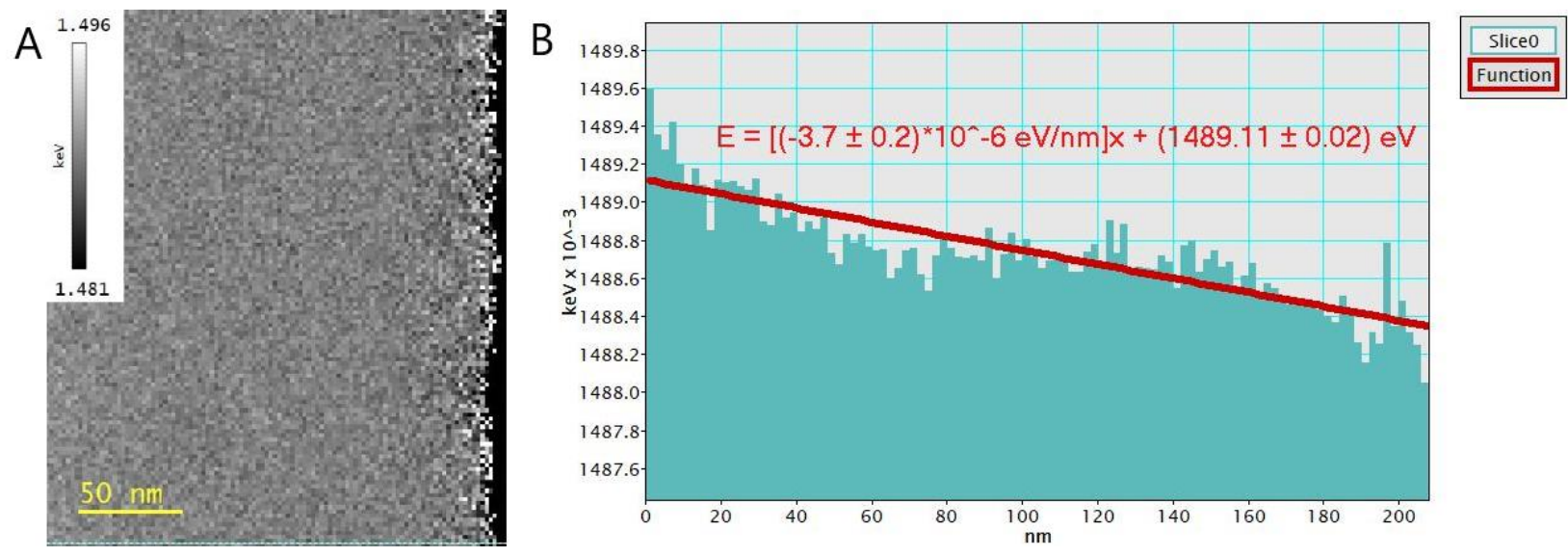

Figure 2. (A) The aluminum Gaussian fit center position map generated from the dataset shown in Fig. 1, thresholded to remove pixels where no x-ray counts are present. Brighter pixel values indicate a higher energy position for the peak at that point. (B) The line profile data (bluegreen), obtained from the position map in (A), drawn horizontally from left to right and averaged along the vertical axis in (A). The fit (red line) shows a decreasing trend from the interior of the sample to its edge, with a net change of $0.8 \mathrm{eV}$.

\section{References}

[1] K Kimoto, T Sekiguchi and T Aoyama, Journal of Electron Microscopy 46 (1997), p. 369-374.

[2] J C Sánchez-López, A Caballero and A Fernández, Journal of the European Ceramic Society 18 (1998), p. 1195-1200.

[3] I Sieber et al., Thin Solid Films 427 (2003), p. 298-302.

[4] R F Egerton and S C Cheng, Ultramicroscopy 55 (1994), p. 43-54.

[5] The data was acquired at the Core Center of Excellence in Nano Imaging (CNI), University of Southern California. This work was supported by National Science Foundation (NSF) Science and Technology Center (STC) award DMR-1548924 (STROBE) and by NSF award DMR-2004897. 